\title{
Authentication in Millimeter-Wave Body-Centric Networks through Wireless Channel Characterization
}

\author{
Nan Zhao, Zhiya Zhang, Masood Ur Rehman, Aifeng Ren, Xiaodong Yang, Jianxun Zhao, Wei Zhao, \\ Binbin Dong
}

\begin{abstract}
Advent of $5 \mathrm{G}$ technologies has ensued in massive growth of body-centric communications (BCC), especially at millimeter-wave frequencies. As a result, the portable/handheld terminals are becoming more and more "intelligent" but not without the cost of being less secure. Improved authentication measures need to be explored, as effective identity authentication is the first level of security in these devices. This paper presents a novel keyless authentication method exploiting wireless channel characteristics. Human palm has distinct transmission coefficient (S21) for each of the users and is used for in-vivo fingerprint identification in this work. A detailed channel modeling using data acquisition from real environment and empirical approach is adopted to evaluate the usability of this method. The results show that this method can provide a secure operation for the millimeter-wave 5G BCCs.
\end{abstract}

Index Terms-Body-centric communications, 5G, Authentication, Security.

\section{INTRODUCTION}

$\mathrm{W}$ IRELESS technologies have seen rapid development and massive expansion in recent years. The global market value of wearable wireless devices alone is estimated to hit $\$ 32$ billion mark by 2020 [1] 5G is the next big step in wireless technologies that would enable ubiquitous portable systems realizing the Internet of Things (IoT). Millimeter-wave frequency range is considered the favorite candidate for the portable $5 \mathrm{G}$ applications for Body-centric Networks and the next generation wireless local access networks (WLANs) due to bandwidth availability, higher throughput and low cost circuit solutions based on advanced CMOS technology [2].

The paper is submitted on 23th, October, 2016. The work was supported in part by National Natural Science Foundation of China (Grant No. 61671349 and 61601338), in part by the Fundamental Research Funds for the Central Universities, in part by the Project Funded by China Postdoctoral Science Foundation, and in part by the Postdoctoral Research Projects Funded in Shaanxi Province.

Nan Zhao, Zhiya Zhang, Aifeng Ren, Xiaodong Yang, Jianxun Zhao, Binbin Dong are with the School of Electronic Engineering, Xidian University, Xi'an, Shaanxi, 710071, China. (Corresponding author: Xiaodong Yang; xdyang@xidian.edu.cn)

M. U. Rehman is with the Centre for Wireless Research, University of Bedfordshire, Luton LU1 3JU, U.K.

Wei Zhao is with the School of Electro-Mechanical Engineering, Xidian University, Xi'an, Shaanxi, 710071, China.
Miniaturized size of the devices at this frequency band also brings in great level of attraction.

Though, high molecular resonance results in higher atmospheric attenuation at millimeter-wave (mmWave) frequencies, at distances lower than $1 \mathrm{~km}$, rain and the atmospheric attenuation on millimeter-wave link at $28-38 \mathrm{GHz}$ is very weak and would not result in communication failure [3]. mmWave communication is therefore, suitable for local or personal communication covering a distance of few meters and has attracted lots of attention from both academia and industry.

Reliability of any wireless communication technology including $5 \mathrm{G}$ has unfathomable dependence on the performance of the antenna and propagation link. Millimeter-wave operation in Body-centric Networks requires the antenna and associated electronics to support increased data rate, larger bandwidth, higher gain, low profile and insensitivity to the human user presence [1]. It necessitates novel antenna designs and efficient channel characterization. These topics have gained high interest from the researchers recently. Liu et al. have discussed the design and working of a $60 \mathrm{GHz}$ chip package for the practical application cycle of 5G [4].

Channel characterization studies are now spanning towards mmWave frequencies [5, 6]. Rappaport has devised a path loss model for millimeter-wave indoor propagation at $73 \mathrm{GHz}$ and $28 \mathrm{GHz}$ [7]. A 3D millimeter-wave statistical channel model for the $5 \mathrm{G}$ applications is proposed in [8]. In [9], a statistical signal receiving model has been proposed for the BCC channel working at mmWave range. Though, these studies have reported preliminary work and promising results in this direction, further research is needed to characterize the propagation environment [10].

Efficient antenna design and channel characterization need understanding of the effects caused by the human body presence in the vicinity. Human body presence deteriorate the performance of wireless devices especially antennas caused by frequency detuning, impedance mismatch and radiation pattern distortions [11]. The radiation performance of the antenna is strongly dependent on the positioning of the device on-body, as well as the user's sex, height and body mass index [12, 13]. It is concluded in [14] that the human body presence at $60 \mathrm{GHz}$ may cause a drop of 18-36 dB in the power level due to the blocking effect.

Human body models are vastly used nowadays to 
accomplish it [1]. The electric properties of human tissues vary with frequency. In order to accurately simulate or create a human body model, the dispersion characteristics of different tissues must be considered. Study of the interaction of the mmWaves and the human body has revealed that the electromagnetic field is almost completely restricted in the skin layer. The main safety problem is the heating of the eyes and the skin due to absorption of mmWave energy and their non-ionizing nature. Therefore, a skin equivalent numerical model is considered to deliver sufficient information on antenna-human body interaction at mmWave frequencies [1]. Moreover, due to the extremely small wavelength of the millimeter-wave frequencies $(1-10 \mathrm{~mm})$ compared to the human body size, it is reasonable to model the human skin as a semi-infinite plane [15]. Due to short range, creeping waves and surface waves have a good potential to contribute to on-body mmWave wireless channel [13]. In addition, more than $95 \%$ body energy absorption can be reduced because of the shielding effect.

The BCCs have major applications in the area of wireless healthcare where physiological attributes of a patient are being monitored and treatment is being provided remotely. This involves transmission and processing of large amount of very personal information of the user by medical devices connected through the internet. It makes this information vulnerable to destruction or eavesdropping that could lead to devastating consequences at personal as well as commercial levels $[1,16]$. Moreover, essential conditions of security in any form of communications are non-falsifiable [17]. It necessitates new security measures to ensure utmost level of personal safety. Identity authentication is the first line of defense against any security infringement. Various techniques have been adopted to establish authenticity of the user in BCCs. Voice prints have been used for security in [18]. However this approach is found to affect privacy of the user and novel identification methods are required to deal with it.

This paper addresses the issue of security in mmWave BCCs by combining the channel characterization and identification. It exploits potential use of the creeping waves and surface waves as a novel keyless method for the authentication purposes. To best of our knowledge, it is a first attempt in this direction.

Following the introduction in this section, the rest of the paper is organized into six sections. Section II characterizes effects of human hand presence on body-centric channel. Section III proposes a novel keyless authentication method for the mmWave body-centric channel based on wireless channel characterization. Conclusion are drawn Section IV.

\section{EFFECT OF HumAN HAND ON BODY-CENTRIC CHANNEL}

Majority of the body-centric applications are portable and handheld where human hand and palm is the integral element devising performance of such devices. Due to the hostile nature of the human body, characterization of the human hand presence at $5 \mathrm{G}$ frequencies; especially mmWave range is of profound importance before using it as a means for security and identification.

According to the Federal Communications Commission
(FCC), the U.S. is being the first country to open high-band spectrum for $5 \mathrm{G}$ networks and technologies [19]. The 5G services are allocated four new frequency bands of $28 \mathrm{GHz}$ (27.5-28.35 GHz), $37 \mathrm{GHz}(37-38.6 \mathrm{GHz}), 39 \mathrm{GHz}$ (38.6-40 $\mathrm{GHz}$ ) and an unlicensed band at $64-71 \mathrm{GHz}$. Since a universally accepted definition of $5 \mathrm{G}$ frequencies is still not available, we have focused our work to study the performance of $5 \mathrm{G}$ applications operating at $28 \mathrm{GHz}(27.5-28.35 \mathrm{GHz})$.

This study establishes the effects of the human hand and palm on mmWave applications through measurements as it offers the advantages of more reliable results due to true replication of a realistic working environment. Moreover, it can avoid the information loss that could take place due to imprecise manufacturing of artificial phantoms and hence, could serve as a benchmark for experimentation done using artificial phantoms. Path loss is estimated in terms of transmission coefficient (S21) measured using Agilent's E8363B Vector Network Analyzer (VNA). Since, flat and grasped postures are the two most common hand actions, two representative test configurations of 'palm flat' and 'palm fist' are considered to evaluate effects of the hand on mmWave wireless channel. Two centre-alligned horn antennas operating at $28 \mathrm{GHz}$ and separated by a distance of $270 \mathrm{~mm}$ are used as the transmitter and receiver to increase the directivity. Fig. 1 shows the measurement setup with key dimensions.

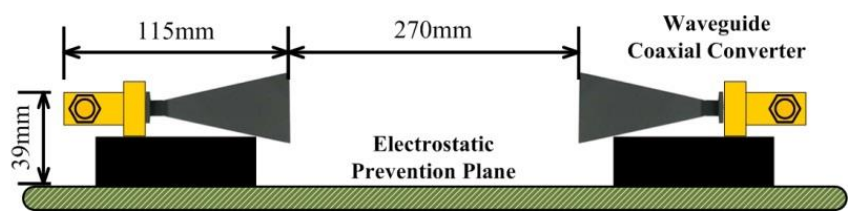

(a)

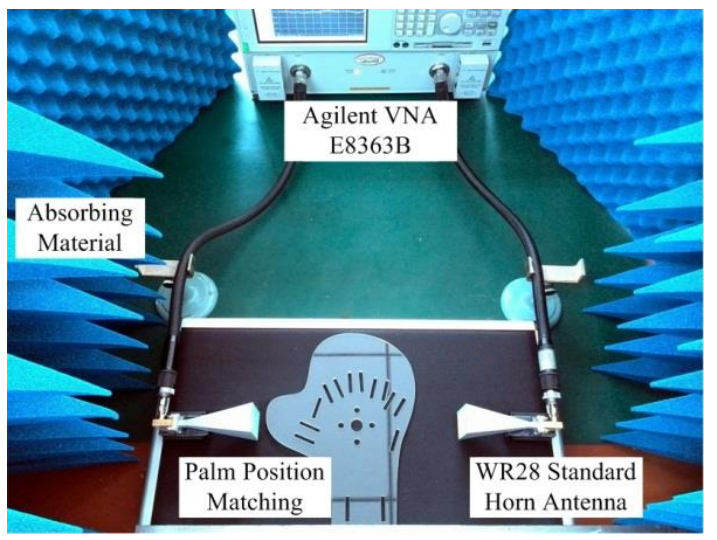

(b)

Fig. 1. Experimental setup showing, (a) key dimensions (b) actual measurement scenario.

The measurement results of the path loss (S21) with curve fitting are summarized in Fig. 2. The results are a clear indication that the influence of the palm on the channel is very significant due to the short wavelength of the mmWave frequency band. The two considered postures of the hand affects the channel distinctly due to varying extents of link blockage. The hand in fist posture introduces relatively larger 
loss (with minimum path loss of $-37 \mathrm{~dB}$ ) as compared to flat posture (with minimum path loss of $-24 \mathrm{~dB}$ ) due to effectively larger blocking area.
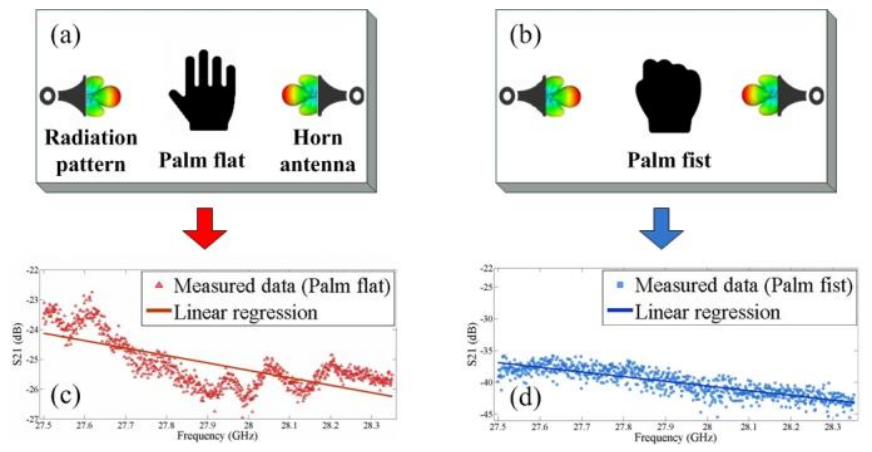

Fig. 2. Measured path loss for on-palm body-centric channel with linear regression (continuous line, $n=-2.49$ or -7.35 ) considering two postures of the hand, (a) flat palm configuration, (b) fisted palm configuration, (c) effects of flat hand on the channel (d) effects of fisted hand on the channel.

To evaluate the stability, repeatability and reliability of the path loss, repeated measurements are carried out using the same human subject at different time instances. The path loss (S21) results for the flat palm and fisted palm configurations are given in Figs. 3 and 4, respectively.

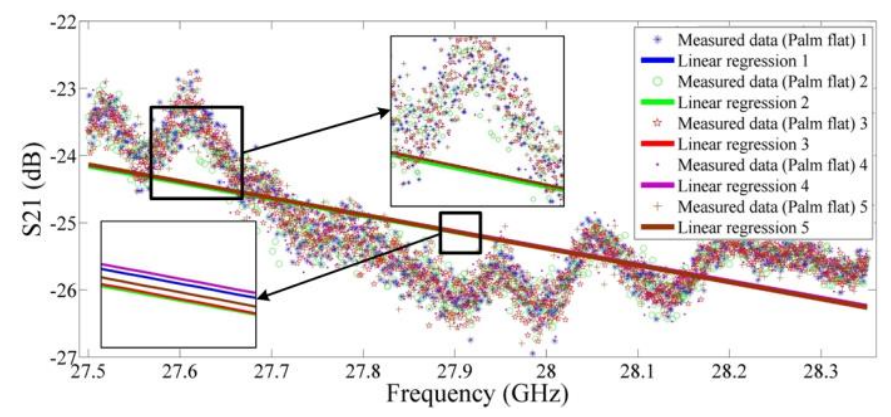

Fig. 3. Path loss measurements with first order linear regression for flat palm hand posture.

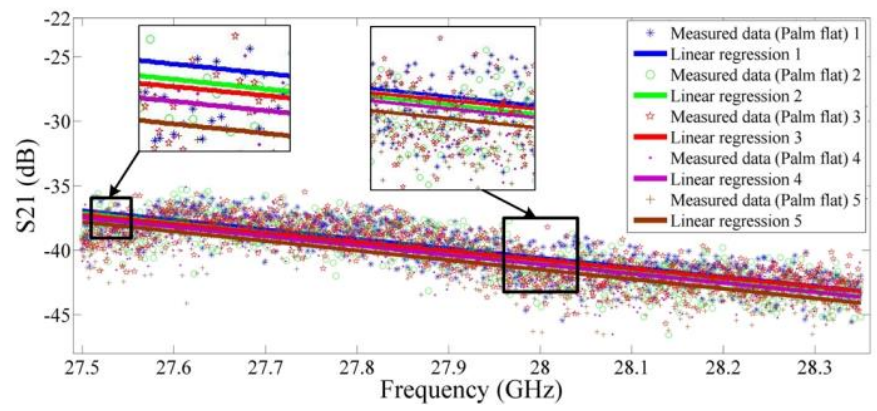

Fig. 4. Path loss measurements with first order linear regression for fisted palm hand posture.

By least squares fitting, we can get the straight line fit in Figs. 3 and 4 , which represents the stability of human body effects on the radio wave channel characteristics. The channel effect factor for a certain hand posture on the wireless channel given in Table I can be calculated considering the following equations:

$$
\sum_{j=1}^{n} x_{i j} \beta_{j}=y_{i},(i=1,2,3 \ldots m)
$$

where $m$ represents the $m$ equations, $n$ represents $n$ unknown $\beta$ parameter, $m>n$, to quantify the equations:

$$
X=\left[\begin{array}{cccc}
x_{11} & x_{12} & \cdots & x_{1 n} \\
x_{21} & x_{22} & \cdots & x_{2 n} \\
\vdots & \vdots & \ddots & \vdots \\
x_{m 1} & x_{m 2} & \ldots & x_{m n}
\end{array}\right], \beta=\left[\begin{array}{c}
\beta_{1} \\
\beta_{2} \\
\vdots \\
\beta_{n}
\end{array}\right], y=\left[\begin{array}{c}
y_{1} \\
y_{2} \\
\vdots \\
y_{n}
\end{array}\right]
$$

Considering the residual sum of squares function:

$$
S(\beta)=\|X \beta-y\|^{2}
$$

when $\beta=\hat{\beta}, S(\beta)$, minimum value can be obtained:

$$
\hat{\beta}=\operatorname{argmin}(S(\beta))
$$

by differential $S(\beta)$ :

$$
\begin{gathered}
X^{T} X \hat{\beta}=X^{T} y \\
\hat{\beta}=\left(X^{T} X\right)^{-1} X^{T} y
\end{gathered}
$$

TABLE I

EFFECT FACTOR OF DIFFERENT HAND POSTURES ON THE WIRELESS CHANNEL

\begin{tabular}{c|c|c|c|c|c}
\hline Posture & \multicolumn{5}{|c}{ Effect factor $1-5$} \\
\hline Palm flat & -2.49 & -2.45 & -2.51 & -2.47 & -2.50 \\
\hline Palm fist & -7.35 & -7.59 & 6.97 & -7.23 & -7.40 \\
\hline
\end{tabular}

It is evident from these results that the same hand posture generates similar profile of the path loss at mmWave frequencies resulting in a stable channel response to that specific posture. These results also show that the effects of the hand presence can be characterized and accounted for in advance through channel measurements. Moreover, since the size of the human body is huge as compared to mmWave wavelength and mmWaves has an exponential decay waveform in the human body, it is being absorbed in the superficial layer of the body making human skin as the primary affected area. It can be explained by analyzing the anatomy of the human skin that can be divided into two layers [15], outer epidermis and dermis layer. The thickness of the epidermis and dermis varies in the range of $0.06-0.1 \mathrm{~mm}$ and $1.2-2.8 \mathrm{~mm}$, respectively. The most superficial layer of the epidermis is called the stratum corneum, with a thickness of 0.012-0.018 mm. Since, mmWave energy decays very rapidly in moist tissues, it penetrates the stratum corneum easily that has low water content of $15-40 \%$. However, it will be absorbed in the deep epidermis and dermis quickly and cannot propagate into body further [20-21]. In [22], mmWave signal penetration depth in the skin has been confirmed to be approximately $3 \mathrm{~mm}$ at $28 \mathrm{GHz}$. The human skin can therefore, be modeled as a semi-infinite plane to the incident mmWaves [15]. Hence, the reflection coefficient from 
arbitrary wave front has a parallel and a perpendicular polarization on the surface of the skin and air:

$$
\begin{aligned}
& R_{\text {parallel }}=\left|\frac{-\varepsilon^{*} \cos \alpha+\sqrt{\varepsilon^{*}-\sin ^{2} \alpha}}{\varepsilon^{*} \cos \alpha+\sqrt{\varepsilon^{*}-\sin ^{2} \alpha}}\right| \\
& R_{\text {perpendicular }}=\left|\frac{\cos \alpha-\sqrt{\varepsilon^{*}-\sin ^{2} \alpha}}{\cos \alpha+\sqrt{\varepsilon^{*}-\sin ^{2} \alpha}}\right|
\end{aligned}
$$

where $\alpha$ is the incident angle, $R_{\text {parallel }}$ and $R_{\text {perpendicular }}$ are power reflection coefficients, $1-R_{\text {parallel }}$ and $1-$ $\mathrm{R}_{\text {perpendicular }}$ are power transmission coefficient.

This discussion leads us to two interesting conclusions. First, that the wireless channel has a distinct signature for varying body configurations and it has unique profile of variation for different postures with good stability at mmWave range as compared to a quasi-static behavior at below $30 \mathrm{MHz}$ frequencies [23]. Second that mmWave offers wide range of changes in the distribution interval and less collision probability making it easier to distinguish between the individuals through channel parameters. These observations combined with the fact that body-centric channels are subject specific and have strong dependence on the user's gender, height, age and body-mass index as well as on-body position of the wireless device $[1,13,24]$, make mmWave channel characteristics a potentially very useful tool for identity authentication. Following section presents a detailed discusses on its viability.

\section{IDENTITY AUTHENTICATION THROUGH MMWAVE CHANNEL CHARACTERIZATION}

Body-centric networks have made remarkable progress in recent years. However, security challenges such as lack of confidence, reliability and data privacy are deterring them from mass acceptance [1]. Common threats include hardware failures, software errors, wireless radio penetration, malware vulnerability, and side-channel attacks. Unlike a communication base station, body-centric communication equipment cannot have a steady stream of power supply. Also, they have to adhere to a small and limited form factor that makes deployment of traditional security measures not feasible.

Researchers have studied use of channel propagation characteristics for security. In [25], tunable capacitors have been used to achieve different bias voltage resulting in varying frequency response. These different frequency responses are then used for device identification. Bhargav et al. have clarified the $\kappa-\mu$ fading channels secrecy capacity [26]. Recently, physical layer security methods based on characterization of the wireless channel are found to be a useful alternative to this ongoing problem. Compared to authentication based on digital key, physical layer authentication is closely related to the communication equipment and communication environment making it extremely difficult to counterfeit. In addition, channel as well as the device would estimate any communication problem and the compensation techniques are the inherent function of both the sender and the receiver. Therefore, authentication based on the physical layer can be accomplished without additional overhead. Notably, the brute force attack, which is a great trouble in traditional cryptography, can be alleviated effectively by the physical layer parameters.

The inherent features of short communication distance and directional antennas of this mmWave wireless channel offers added benefits for the security and authentication. Due to the transmission limitations, the receiver needs to be located at closer distances. It removes the threat of eavesdropping by far located attackers. Furthermore, mmWave communications use highly directional antennas with narrow beams to overcome high path loss. It will result in low signal-to-noise ratio at the attackers end limiting his ability to snoop the information. This section focuses on exploiting radio and propagation characteristics of mmWave channel at $28 \mathrm{GHz}$ to implement a new authentication method.

\section{A. Measurement Set-up}

The existing wireless equipment cannot achieve acceptable level of authentication in the actual environment. It is therefore, necessary to explore new ways for certification. This section introduces the experimental design of identity authentication through channel measurements. S21 is employed to characterize the wireless channel. We need to consider a number of factors while designing the experiment due to unavailability of the relevant information at $28 \mathrm{GHz}$ frequency range.

In [27], it has been concluded that achieving a non-line-of-sight on-body communication channel at mmWave frequencies is very difficult because of the typical shadow fading. A face-to-face system structure is found to be useful in such a situation [28]. The penetration of the mmWave is restricted to the human skin [20-21]. The horn antennas are a proven efficient choice to measure wireless channel at $28 \mathrm{GHz}$ body-centric networks due to higher directionality interference minimization [29, 30]. Based on these findings, we have designed our experiment to have a face-to-face system structure with directional horn antennas as the transmitter and receiver.

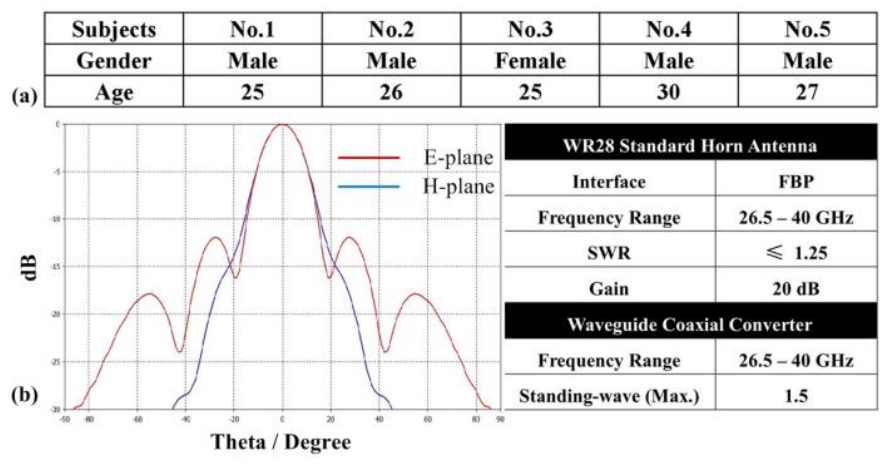

Fig. 5. Human subject age and gender characteristics with antenna radiation parameters, (a) subject characteristics, (b) horn antenna and waveguide coaxial converter parameters.

The experimental set-up is similar as shown in Fig. 1. Two WR28 horn antennas facing each other and the Agilent E8363B VNA are used to acquire S21 information for the on-body channel with varying hand postures. The horn antennas have a gain of $20 \mathrm{~dB}$. They are separated by a distance of $270 \mathrm{~mm}$ and placed at a height of $39 \mathrm{~mm}$. The antennas are connected to the 
VNA using $70 \mathrm{~cm}$ long coaxial cables and waveguide-to-coaxial converters.

To avoid possible device and system errors in the experiment, conventional procedure of calibration is performed for the VNA. Losses in the cables and converters are also measured and taken into account via calibration. In order to prevent electrostatic damage to the instrument, all experiments are conducted on the anti-static test-bed.

Microwave-absorbing material is placed in the surrounding to reduce interference. Fig. 5 shows the radiation parameters of the horn antennas and the converters.

Five human subjects of roughly same physique and size have been used in this study. Ten sets of data have been recorded for each of the human subject. Authentication has been achieved through observing a distinct channel profile, a unique in-vivo fingerprint, for each of the human subjects.

\section{B. Results and Analysis}
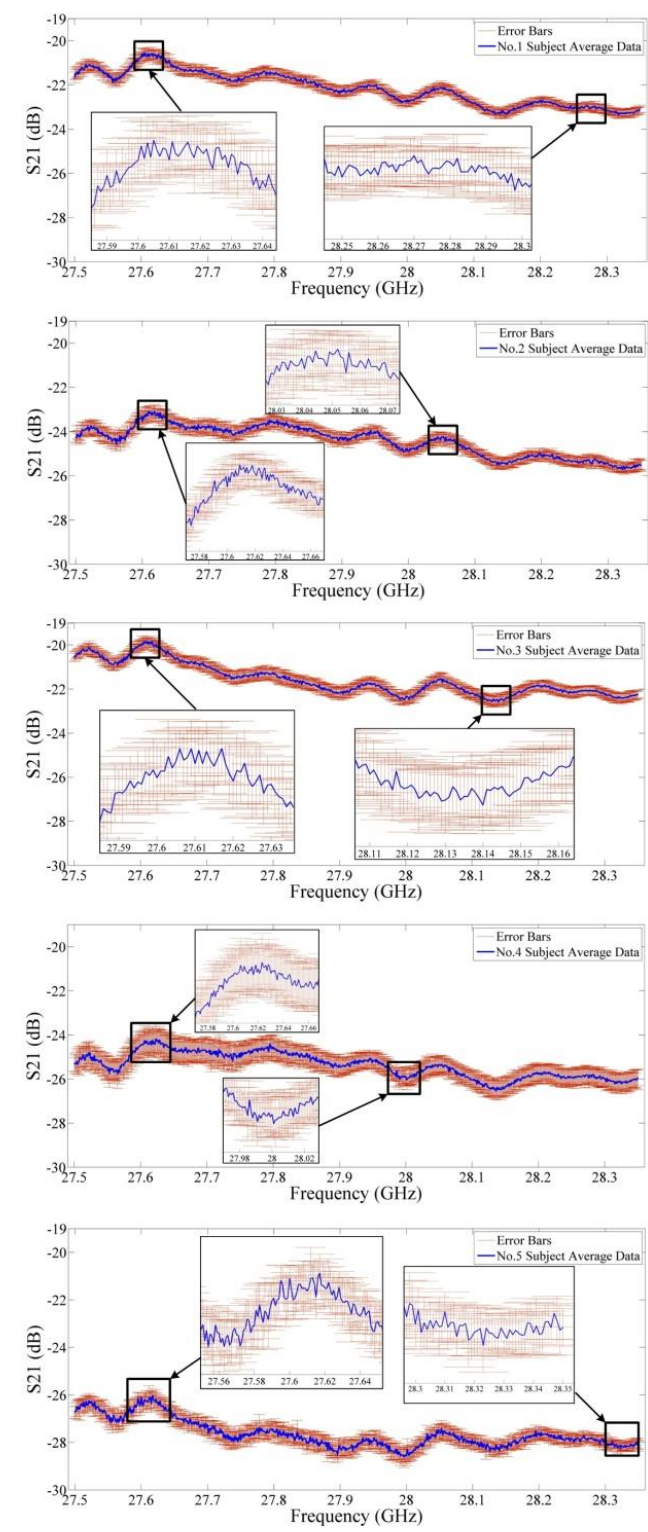

Fig. 6. In-vivo channel profile of five human subjectsfor flat palm configuration (continuous line indicates mean value and error bar represents standard deviation).
The measurement results of the in-vivo channel profile with human hand presence associated to the five human subjects are shown in Fig. 6. The blue line indicates the average curve of the 10 measured data sets while the red bars illustrate standard deviation. Both parameters show that the results for the five test subjects have significant differences and unique profile enabling them to be clearly distinguishable even without a need of post-processing. The results also exhibit good stability through high repeatability of the measurements. Moreover, different test subjects have produced different power levels due to slightly different electric properties of the hand tissues. These observations are in good agreement with the previous studies.

To investigate the strength of the proposed physical layer authentication method, the raw data measurements of the channel profile for the five test subjects are plotted and compared in Fig. 7. Correlation coefficients between these S21 sequences and effect factor are then calculated and summarized in Table II.

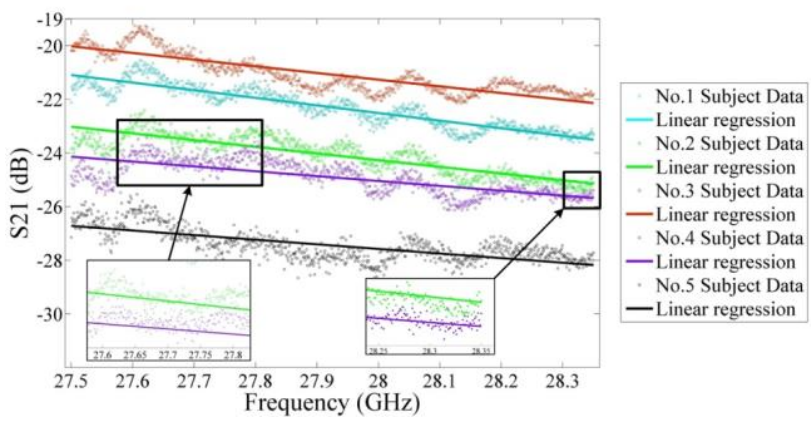

Fig. 7. In-vivo channel response of five participants, raw data (scatter plot), linear fitting (continuous line).

TABLE II

CORRELATION COEFFICIENTS FOR IN-VIVO CHANNEL RESPONSE OF THE FIVE TEST SUBJECTS (FIG. 6) AND EFFECT FACTOR IN FIG. 7

Correlation coefficient of each other in Fig. 7

\begin{tabular}{c|c|c|c|c|c} 
Subjects & 1 & 2 & 3 & 4 & 5 \\
\hline 1 & 1 & 0.9561 & 0.9192 & 0.9338 & 0.7926 \\
\hline 2 & 0.9561 & 1 & 0.7807 & 0.9534 & 0.6170 \\
\hline 3 & 0.9192 & 0.7807 & 1 & 0.7868 & 0.9544 \\
\hline 4 & 0.9338 & 0.9534 & 0.7868 & 1 & 0.6323 \\
\hline 5 & 0.7926 & 0.6170 & 0.9544 & 0.6323 & 1 \\
\hline \multicolumn{6}{|c}{ Effect factor 1-5 in Fig.8 } \\
\hline $\mathrm{n}$ & -2.84 & -2.50 & -2.49 & -1.81 & -1.72 \\
\hline
\end{tabular}

Comparison of the plots in Fig. 7 shows that there is a good discrimination between the five data sets associated to the five test subjects. The magnified observation of the data sets further establishes that the five test subjects influences the wireless channel differently with a distinct S21 sequence, even in the areas that seem similar at first look. The effect factor shown in terms of the linear fitting indicates that despite having different 
power levels, overall channel response is consistent and stable for different users. These observations are also in agreement with the calculations presented in Table II. It is quite certain from this discussion that the S21 data in this body-centric wireless communication link is subject specific and stable. It can therefore, be used as a good means to identify the right user and provide authentication.
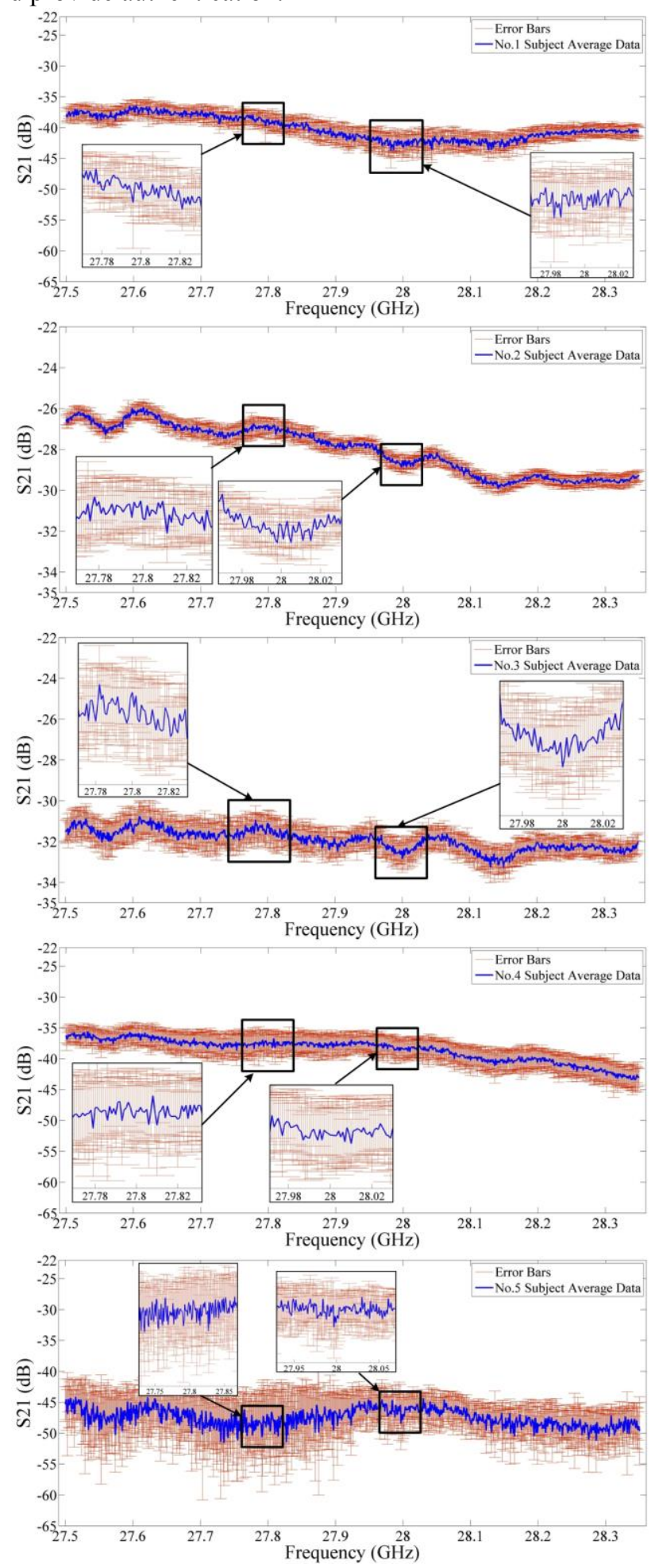

Fig. 8. In-vivo channel profile of five human subjects for fisted palm configuration (continuous line indicates mean value and error bar represents standard deviation).
Through Fig. 6 (palm flat) and Fig.8 (palm fist), it can be found that, the error bars are almost close to the S21 data mean line, this shows that the mmWave body centric wireless channel characterization has good measurement repeatability in the above scenarios and for different users.

Moreover, it can also be seen that age and gender of the subjects, as given in Fig. 5(a), have little impact on the experimental results. Although small, the S21 characteristics are mainly affected due to different physical attributes of an individual's palm like size and shape. The skin layer also brings slight changes to mmWave propagation.

The fisting action (movement of hand from flat palm to closed palm) results in larger error as compared to the static condition of fisted palm. This is evident from the larger variation in the error bars in measurement data for subject No. 5 in Fig. 8. It is therefore concluded that the mmWave channel information can result in better identification levels with good distinction and repeatability for bare palm and static hand configurations. Repeatability is also evident from the results given in Figs. 6 and 8 where same human subjects are used to collect data with only a small level of discrepancies. Furthermore, these results also indicate that flat palm and fisted palm only slightly changes the results ensuring high level of repeatability.

As a new biometric feature proposed from the perspective of antenna and propagation, this paper focuses on pointing out its feasibility and usefulness compared to the traditional bio-recognition methods such as fingerprint and iris.

Its advantages include robustness due to flexible nature of the wireless channel. Moreover, the three-dimensional propagation space of the wireless signal enables this authentication method to extend the general two-dimensional biometrics into three or even four dimensions (three spatial dimensions plus the time dimension). These higher dimensions can provide richer features, more reliable and more flexible authentication. On top of that, this authentication method for the mmWave body-centric systems relies on the channel feature extraction using S21 which is a typical part of practical communication applications. Hence, it needs almost no additional hardware making it extremely cost-effective.

Despite offering high level of security with minimum overhead, the keyless physical authentication is only possible if the assailant cannot imitate the legitimate channel. Variations in the antenna radiation pattern due to proximity of the human body could also limit its applicability. Moreover, different antenna types may also affect the data acquisition to a small extent. Though mmWave body-centric channels are less affected by these two degrading factors, work to furnish a more realistic radiation model is needed to completely rectify these problems.

\section{CONCLUSION}

The next generation of consumer wireless systems $(5 \mathrm{G})$ is on the horizon. This system would be a key part of the mmWave body-centric communications. Authentication and security issues are currently one of the major issues faced by the body-centric networks. Very limited work is available on this 
topic at present, especially for the systems operating at $28 \mathrm{GHz}$ frequency range.

A novel keyless authentication method for the $28 \mathrm{GHz}$ mmWave body-centric systems has been proposed. This technique is based on the wireless channel response to the presence of the human hand. As a prelude to the authentication method, the subject specificity, repeatability and stability of the $28 \mathrm{GHz}$ wireless channel have been established through S21 measurements. The experimental measurements and numerical analysis has shown that the channel reacts quite discriminatorily to different hand postures and different users. It has been shown that this channel characteristic can be used effectively to identify the right user and authenticate the data usage. Moreover, this physical layer based security in body-centric communications can be accomplished without any additional overhead. It makes it a suitable candidate for short range and directive mmWave communication links. This study is first attempt in physical layer mmWave channel security and presents very encouraging results. Work is in progress to further evaluate its usefulness in order to assess the False Acceptance Rate (FAR) and False Rejection Rate (FRR) scenarios.

\section{REFERENCES}

[1] Abbasi, Q. H.; Ur Rehman, M.; Qaraqe, K. and Alomainy, A. (Ed.), "Advances in Body-Centric Wireless Communication: Applications and State-of-the-art", the IET (UK), 2016, ISBN: 978-1849199896.

[2] N. Chahat, C. Leduc, M. Zhadobov and R. Sauleau, "Antennas and interaction with the body for body-centric wireless communications at millimeter-waves," Antennas and Propagation (EuCAP), 2013 7th European Conference on, Gothenburg, 2013, pp. 772-775.

[3] T. S. Rappaport et al., "Millimeter Wave Mobile Communications for 5G Cellular: It Will Work!," in IEEE Access, vol. 1, no. , pp. 335-349, 2013.

[4] D. Liu and Y. P. Zhang, "Integration of Array Antennas in Chip Package for $60-\mathrm{GHz}$ Radios," in Proceedings of the IEEE, vol. 100, no. 7, pp. 2364-2371, July 2012.

[5] A. Brizzi, A. Pellegrini, L. Zhang and Y. Hao, "Statistical Path-Loss Model for On-Body Communications at $94 \mathrm{GHz}$," in IEEE Transactions on Antennas and Propagation, vol. 61, no. 11, pp. 5744-5753, Nov. 2013.

[6] A. Brizzi and Y. Hao, "Experimental investigation of the effect of clothes on the propagation of millimeter waves over the human trunk," Wireless Symposium (IWS), 2013 IEEE International, Beijing, 2013, pp. 1-4.

[7] G. R. Maccartney, T. S. Rappaport, S. Sun and S. Deng, "Indoor Office Wideband Millimeter-Wave Propagation Measurements and Channel Models at 28 and $73 \mathrm{GHz}$ for Ultra-Dense 5G Wireless Networks," in IEEE Access, vol. 3, no. , pp. 2388-2424, 2015.

[8] M. K. Samimi and T. S. Rappaport, "3-D Millimeter-Wave Statistical Channel Model for 5G Wireless System Design," in IEEE Transactions on Microwave Theory and Techniques, vol. 64, no. 7, pp. 2207-2225, July 2016.

[9] S. L. Cotton, "A Statistical Model for Shadowed Body-Centric Communications Channels: Theory and Validation," in IEEE Transactions on Antennas and Propagation, vol. 62, no. 3, pp. 1416-1424, March 2014.

[10] Y. Hao, A. Brizzi, R. Foster, M. Munoz, A. Pellegrini and T. Yilmaz, "Antennas and propagation for body-centric wireless communications: Current status, applications and future trend," Electromagnetics; Applications and Student Innovation (iWEM), 2012 IEEE International Workshop on, Chengdu, Sichuan, 2012, pp. 1-2.

[11] M. O. Munoz, R. Foster and Y. Hao, "On-Body Channel Measurement Using Wireless Sensors," in IEEE Transactions on Antennas and Propagation, vol. 60, no. 7, pp. 3397-3406, July 2012.

[12] A. Sani, A. Alomainy and Y. Hao, "Numerical Characterization and Link Budget Evaluation of Wireless Implants Considering Different Digital Human Phantoms," in IEEE Transactions on Microwave Theory and Techniques, vol. 57, no. 10, pp. 2605-2613, Oct. 2009.
[13] M. U. Rehman, Y. Gao, X. Chen, C. Parini and Z. Ying, "Environment effects and system performance characterisation of GPS antennas for mobile terminals," in Electronics Letters, vol. 45, no. 5, pp. 243-244, February 262009.

[14] Y. P. Zhang and D. Liu, "Antenna-on-Chip and Antenna-in-Package Solutions to Highly Integrated Millimeter-Wave Devices for Wireless Communications," in IEEE Transactions on Antennas and Propagation, vol. 57 , no. 10 , pp. $2830-2841$, Oct. 2009.

[15] T. Wu, T. S. Rappaport and C. M. Collins, "Safe for Generations to Come: Considerations of Safety for Millimeter Waves in Wireless Communications," in IEEE Microwave Magazine, vol. 16, no. 2, pp. 65-84, March 2015.

[16] Clery, Daniel, "Could your pacemaker be hackable?" in Science, 347.6221:499-499, 2015.

[17] Herley, Cormac, "Unfalsifiability of security claims," in Proceedings of the National Academy of Sciences, 2016.

[18] Shultz, David, "When your voice betrays you," in Science 347.6221:494-494, 2015

[19] Fact Sheet: Spectrum Frontiers Proposal To Identify, Open Up Vast Amounts Of New High-Band Spectrum For Next Generation (5g) Wireless Broadband. (Jun 23, 2016). Available: https://apps.fcc.gov/edocs_public/attachmatch/DOC-339990A1.pdf

[20] A. R. Guraliuc, M. Zhadobov, G. Valerio, N. Chahat and R. Sauleau, "Effect of Textile on the Propagation Along the Body at $60 \mathrm{GHz}$," in IEEE Transactions on Antennas and Propagation, vol. 62, no. 3, pp. 1489-1494, March 2014.

[21] A. R. Guraliuc, M. Zhadobov, G. Valerio, N. Chahat and R. Sauleau, "Characterization of the body-centric propagation channel at $60 \mathrm{GHz}$ in the presence of textiles," The 8th European Conference on Antennas and Propagation (EuCAP 2014), The Hague, 2014, pp. 3575-3577.

[22] W. Hong, K. H. Baek, Y. Lee, Y. Kim and S. T. Ko, "Study and prototyping of practically large-scale mmWave antenna systems for $5 \mathrm{G}$ cellular devices," in IEEE Communications Magazine, vol. 52, no. 9, pp. 63-69, September 2014.

[23] N. Haga, K. Saito, M. Takahashi and K. Ito, "Equivalent Circuit of Intrabody Communication Channels Inducing Conduction Currents Inside the Human Body," in IEEE Transactions on Antennas and Propagation, vol. 61, no. 5, pp. 2807-2816, May 2013.

[24] P. S. Hall, Y. Hao and S. L. Cotton, "Advances in antennas and propagation for body centric wireless communications," Proceedings of the Fourth European Conference on Antennas and Propagation, Barcelona, Spain, 2010, pp. 1-7.

[25] A. Mahmood and M. A. Jensen, "Data-dependent transmitter fingerprints for radio authentication," 2014 IEEE Radio and Wireless Symposium (RWS), Newport Beach, CA, 2014, pp. 265-267.

[26] N. Bhargav, S. L. Cotton and D. E. Simmons, "Secrecy Capacity Analysis Over k - u Fading Channels: Theory and Applications," in IEEE Transactions on Communications, vol. 64, no. 7, pp. 3011-3024, July 2016.

[27] N. Chahat, G. Valerio, M. Zhadobov and R. Sauleau, "On-Body Propagation at $60 \mathrm{GHz}$," in IEEE Transactions on Antennas and Propagation, vol. 61, no. 4, pp. 1876-1888, April 2013.

[28] L. Petrillo, T. Mavridis, J. Sarrazin, A. Benlarbi-Delaï and P. De Doncker, "Statistical On-Body Measurement Results at $60 \mathrm{GHz}$," in IEEE Transactions on Antennas and Propagation, vol. 63, no. 1, pp. 400-403, Jan. 2015.

[29] T. Zwick, C. Baks, U. R. Pfeiffer, Duixian Liu and B. P. Gaucher, "Probe based MMW antenna measurement setup," Antennas and Propagation Society International Symposium, 2004. IEEE, 2004, pp. 747-750 Vol.1.

[30] X. Wu, Y. I. Nechayev, C. C. Constantinou and P. S. Hall, "Interuser Interference in Adjacent Wireless Body Area Networks," in IEEE Transactions on Antennas and Propagation, vol. 63, no. 10, pp. 4496-4504, Oct. 2015. 


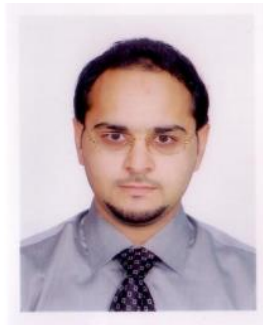

Masood Ur Rehman (SM'16) received the B.Sc. degree in electronics and telecommunication engineering from University of Engineering and Technology, Lahore, Pakistan in 2004 and the M.Sc. and Ph.D. degrees in electronic engineering from Queen Mary University of London, London, UK, in 2006 and 2010, respectively. He worked at Queen Mary University of London as a postdoctoral research assistant till 2012 before joining the Centre for Wireless Research at University of Bedfordshire, University Square, Luton, UK, as a lecturer. His research interests include compact antenna design, radiowave propagation and channel characterization, satellite navigation system antennas in cluttered environment, antenna interaction with human body, body-centric wireless networks and sensors, remote health care technology, mmWave and nano communications for body-centric networks and body-to-body communications. He has worked on a number of projects supported by industrial partners and research councils. He has contributed to a patent and authored/coauthored 3 books, 5 book chapters and more than 55 technical articles in leading journals and peer reviewed conferences. Dr. Ur Rehman is a Fellow of the Higher Education Academy (UK), a member of the IET and part of the technical program committees and organizing committees of several international conferences, workshops and special sessions. He also serves as an associate editor of IEEE Access and reviewer for book publishers, IEEE conferences and leading journals.

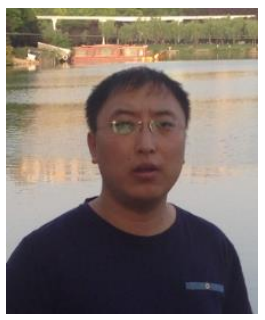

Xiaodong Yang (M'12) He has published over 30 peer-review journal and conference papers, including IEEE Transactions and Letters. His current research interests include body area networks, biomedical communications, antennas and propagation, physical layer security, etc. Dr. Yang received the Young Scientist Award from the International Union of Radio Science in 2014. He is an Active Reviewer for IEEE Transactions and Letters. 